Año 19, № 50 San Salvador, El Salvador, Centroamérica. Revista Semestral julio-diciembre 2019

YEAR 19, N 50 SAN SALVADOR, El SALVADOR, CENTRAL AMERICA. SEMESTRAL JOURNAL JLUIO- DICIEMBRE 2019

\title{
Las tierras con vocación cafetalera y el Martinato 1933: génesis del municipio del Congo, departamento de Santa Ana
}

\author{
The lands with a coffee vocation and the Martinato 1933: Genesis of the \\ Municipality of Congo, Department of Santa Ana
}

\author{
Luis Alberto Martínez Marín \\ Antropólogo sociocultural Universidad de El Salvador \\ Imartinezmarin4@gmail.com \\ Recibido: 7 de abril de 2019 \\ Aprobado: 29 de noviembre de 2019 \\ DOI: 10.5377/ryr.v50i50.9093
}

\section{RESUMEN}

El trabajo está encaminado a develar cómo "la vocación cafetalera" de las tierras altas del departamento de Santa Ana, específicamente los municipios de Coatepeque, y parte de lo que hoy constituye el municipio de E1 Congo, 1933; que se escindió del primero, fue donde se extendió la frontera agrícola santaneca. Además, quiere mostrar parte de una política clientelar que forjó y mantuvo la era del General Maximiliano Hernández Martínez (1931-1944), y coadyuvó al surgimiento del municipio de El Congo, en 1933.

Palabras clave: Coatepeque, producción de café, creación del municipio de El Congo.

\section{ABSTRACT}

The work is aimed to reveal as "the coffee vocation", from the highlands of the Department of Santa Ana, specifically the municipalities of Coatepeque, and part of what is now the municipality of El Congo 1933; hat was split from the first one, was where the agricultural frontier of Santa Ana was extended, but, it wants to show part of a clientelist policy that forged and maintained the era of General Maximiliano Hernández Martinez (1931-1944), and contributed to the emergence of the municipality of El Congo in 1933.

Key words: Coatepeque, coffee production, creation of the municipality of El Congo. 


\section{Introducción}

Según, la estadística general de la República de E1 Salvador, 1858, Coatepeque era un pueblo con 2,436 habitantes (...) en terreno firme, pero algo quebrado (...) dista de Santa Ana tres leguas al suroeste (...) la ocupación dominante de los vecinos es la agricultura (...) la cosecha dominante entre pocos años será el café (1990, p.75).

La anterior cita si bien, brinda una idea sobre el quehacer de esta localidad, el último párrafo, nos sitúa en la perspectiva del impacto que tendrá en la vida nacional, lo que algunos estudiosos dieron en llamar la república cafetalera salvadoreña, en pueblos indígenas como Coatepeque.

La cercanía a Santa Ana, centro de poder económico y político, consolidó a los demás municipios como "satélite" de los intereses y perspectivas de la clase gobernante-comerciante que se legitimó en la gran urbe; y cuya primacía de proyecto nacional estatal disputaba con San Salvador a comienzos del siglo XX.

Para llevar a cabo la presente investigación de corte descriptivo, se utilizaron fuentes bibliográficas y hemerográficas que permitieron un acercamiento histórico-antropológico que pretende ver más allá de lo meramente económico, y utiliza el concepto de sistema clientelar ${ }^{1}$ para develar las situaciones particulares en la génesis del municipio de El Congo, y la era del General Maximiliano Hernández Martínez y sus clientes.

1 Sistema clientelar: Consiste en una red, a nivel nacional de relaciones patrón-cliente, en la cual los actores, a nivel local regional o nacional estaban relacionados con red de jerarquía. Las decisiones se toman en la última instancia de la cúspide a nivel nacional, pero las redes locales o regionales ejercen poder de negociación. (Ching Erick, 2007, p.140).

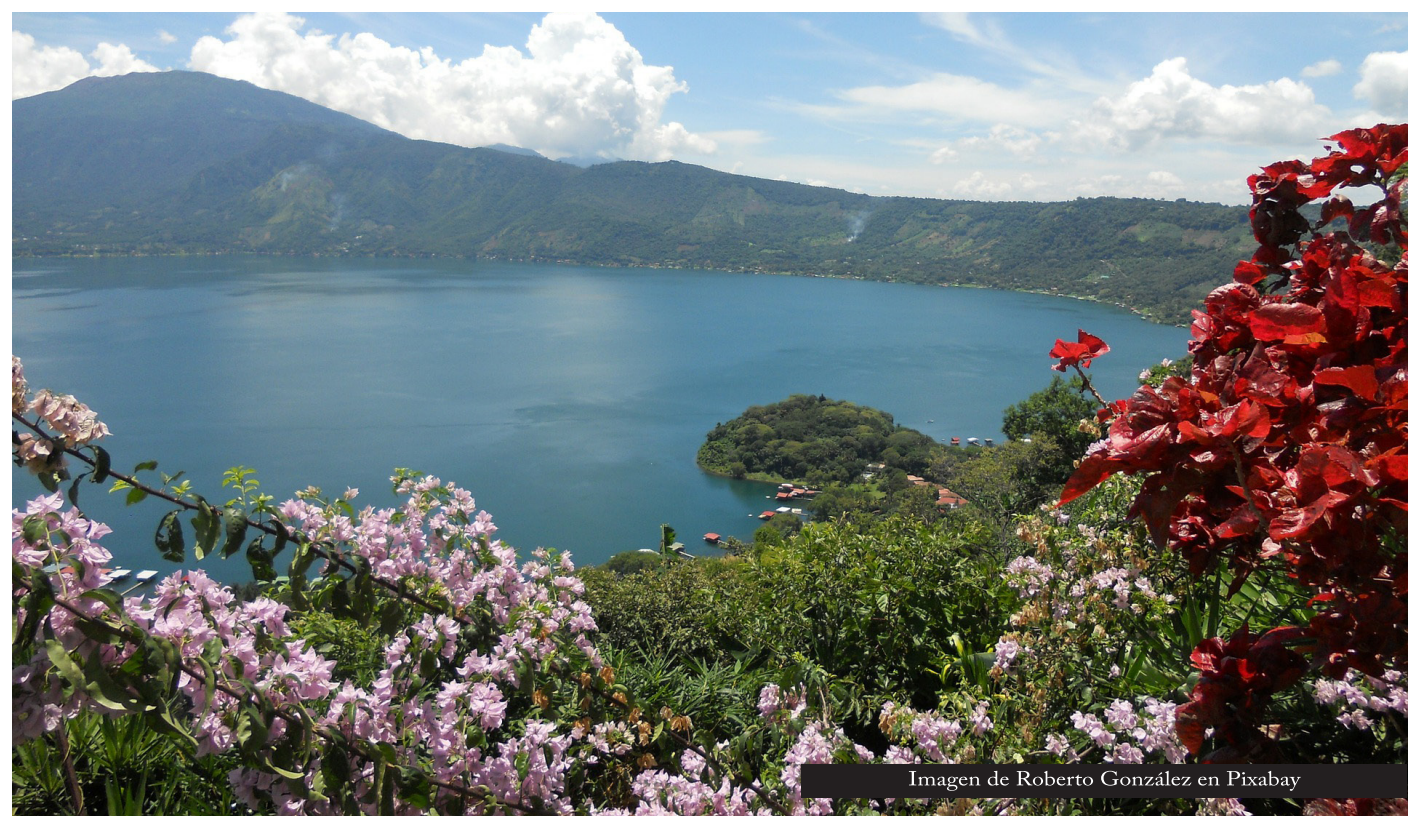




\section{Coatepeque: la frontera agrícola de Santa Ana}

Coatepeque a principios del siglo XX, hasta 1917 cuando fue declarada ciudad, era una villa con amplias y heterogéneas regiones, de aproximadamente $218 \mathrm{~km}^{2}$, con "19 valles y cuatro barrios: Jesús, Calvario, San José y centro" (Barberena, 1998, p.145), un lago con zonas privadas donde se recreaban las autoridades de la época; el mismo Hernández Martínez tenía rancho en las riberas del lago de Coatepeque y todo esto hizo difícil el ejercicio del poder político que emanaba de la municipalidad (se verifica lo anterior en $2014^{2}$ ). Fue delimitada su frontera con Santa Ana, mientras otros segmentos territoriales aún esperan delimitación.

Además, Santa Ana, extendió su frontera agrícola hacia Coatepeque, que en parte fue en: "la ocupación de baldíos y las compras de lotes ejidales y comunales por empresarios de otras localidades -ligados a intereses económicos de quiénes gobernaban Santa Ana- (Lauria, 2003, p. 222); por ejemplo, "un baldío alrededor del lago de Coatepeque fue convertido en fincas de café y sitio de veraneo para la élite de la ciudad" (Lauria, 2003, p. 224).

En cuanto a los procesos económicos es de señalar cómo esta región se "activa" con el auge del cultivo del café y la introducción del ferrocarril, en las primeras décadas del siglo XX, lo que trae una dinámica que es propia a este proceso. Sus tierras son aptas para tal cultivo, lo que sin duda, puso presión sobre sectores como el indígena y sus tierras; por ejemplo, muy tempranamente en 1860: "Barrios (presidente) intervino en una disputa legal entre la comunidad indígena

2 Según Diario Oficial decreto No 770 de agosto de 2014. de Coatepeque y un interesado que deseaba inscribir tierras conocidas como «siete príncipes» como baldías" (Menéndez citado en Lauria, 2003, p.87).

Hechos como el citado anteriormente, en el que don Dolores Vides -familiar de cafetalerosreclamó tierras comunales y ejidales, ocupadas por los indígenas de Coatepeque, alegando que se trataba de terrenos "baldíos" se acentuaron cuando entraron en vigencia las leyes de extinción de ejidos y tierras comunales ${ }^{3}$; pues, la normativa establecía que "el título para tener derecho a cultivar lo que se quisiera y en la cantidad de terreno que se deseara era de $\$ 3.00$, por ley se debía cultivar café" (Montes, 1986, p.55) Así mismo, se dividieron a las comunidades, generando conflictos entre estas y otros grupos o el mismo Gobierno.

Coatepeque fue el municipio que experimentó mayor crecimiento de la producción de quintales de café, a finales del siglo XIX

\section{Cuadro n. ${ }^{\circ} 1$}

Producción cafetalera

\begin{tabular}{|l|l|l|}
\hline 1858 & $3000 \mathrm{qq}$ & 7 haciendas \\
\hline 1876 & $12000 \mathrm{qq}$ & 161 fincas de -11 hectáreas \\
\hline
\end{tabular}

Fuente: Elaboración propia en base a Estadística general de la República 1858-1861.

La ideología de corte capitalista que se instala en Santa Ana va a permitir las condiciones para que surja y pueda maniobrar un estrato que se

3 La ley de extinción de tierras comunales y ejidales (1881) fue redactada por Teodoro Moreno, cafetalero, funcionario público, la ley empieza así: La existencia de tierras bajo la propiedad de las comunidades impide el desarrollo agrícola, estorba la circulación de la riqueza y debilita los lazos familiares y la independencia del individuo... (Browing 1998, p- 338). 
presenta como "comerciante", civiles algunos, otros militares, funcionarios, son burgueses agrarios que representan los anhelos e intereses de los grandes capitalistas de la urbe santaneca ${ }^{4}$, estos fortalecen un sistema caudillo-patrón/ clientelar que permite llevar exitosamente el proceso económico y las alianzas locales que consolidan al gobernante pero que dividen las comunidades y del cual se verán recompensados estos comerciantes con algunas concesiones de poder o terrenos a bajos precios. Pero, en lo político, es quizás donde enfrentan la mayor oposición de grupos que se organizan y que terminan enfrentándose pero que luego, son aplastados, ellos se consagran como agitadores y comunistas.

\section{Los focos de inestabilidad}

En la historia particular de Santa Ana, el sistema político durante el siglo XIX, "institucionalizó" el sistema de patrón-clientela en el cual comunidades y "líderes" eran recompensados por apoyar la causa del caudillo que pretendía mantenerse en el poder: "En 1871, el nuevo Gobierno de (Santiago González) compensó a los volcaneños por ayudar en la derrota de Dueñas, al reconocer su posesión de hecho de los terrenos reclamados" (Lauria, 2003, p. 192).

Pero este sistema de clientelas subsistió con algunos matices entrado el siglo XX. En la creación del municipio de El Congo, en 1933;

\footnotetext{
4 Según Portillo (2006) había diversas formas de relaciones sociales cafetaleras en la Santa Ana del siglo XIX, en la cima estaba la burguesía-industrial-comercial, que se enriquece del beneficiado y la exportación de café. De su cultivo se encargan una mayoría de extranjeros. Luego, una burguesía agraria-cafetalera que no alcanza el rubro de la exportación y por último, un campesinado cafetalero (p. 152).
}

época del llamado "Martinato", caracterizada por el control y represión debido al levantamiento indígena-campesino de 1932, en Izalco y otros pueblos del occidente. En esta época se intenta identificar a la población y se dicta una ley xenófoba de extranjería que decía: "Se prohíbe la entrada al país, a los extranjeros comprendidos en uno o más de los casos siguientes: a los de raza negra; a los malayos y a los gitanos (...) el ingreso al país de nuevos inmigrantes originarios de Arabia, Líbano, Siria, Palestina, o Turquía” (Gómez, 2013, p. 129).

Sin embargo, hay tres sucesos que nos describen el contexto social y político de la creación del municipio de El Congo, en 1930. En primer lugar, las condiciones de la vida en lo rural estaban tensas, había huelgas y represión estatal: las protestas de los campesinos se convirtieron entonces de acciones aisladas en la zona occidental de El Salvador a movimientos huelguísticos de fuertes e incalculables proporciones. Sorprendido por las crisis, el Gobierno de Arturo Araujo no estaba preparado para contener la ola de anarquía incipiente (Cuenca citado en Anderson, 2006, p.145). Así, el 2 de diciembre de 1931, se dio un golpe de Estado, que permitió la llegada al poder del General Maximiliano Hernández Martínez, quien fuera el vicepresidente y ministro de Guerra del depuesto presidente Araujo, por 14 años.

El otro suceso es la aguda crisis internacional que repercute en los precios del café, en agosto de 1929, lo que contribuyó a la inestabilidad de las condiciones laborales y salariales de los trabajadores de las fincas de café; entre otros efectos.

Lo que se puede advertir es un contexto de inestabilidad política y de regiones sin control 
estatal, muchas de las cuales, por su geografía, ofrecían las condiciones para mantenerse fuera de control y algunas de estas se convertirían luego en los cantones del nuevo municipio de $\mathrm{El}$ Congo, en 1933.

Por ejemplo, en 1930, la hacienda "La Presa", propiedad de Claudia Borbón, viuda de Guirola, situada casi en el límite entre los departamentos de Sonsonate y Santa Ana:

La Guardia Nacional desahució a 345 familias de colonos en medio de una tormenta, manifestando que el sindicato había exigido la expropiación y la subsiguiente división de los terrenos para los colonos. En realidad, el sindicato había amenazado con una huelga en demanda de mejores salarios y la exoneración del pago del agua. Eventualmente, se le permitió a muchas de las familias regresar a sus hogares, en tanto los cuatro dirigentes sindicales fueron encarcelados, ocasionando el desmoronamiento del sindicato (Lauria y Gould, 2005, p. 328)

Antes de los sucesos de 1932 se sabe muy poco acerca de lo que aconteció en la zona sur de Santa Ana, limítrofe con el departamento de Sonsonate, y en la misma cabecera santaneca, pero algunos escritos denunciaban que en la hacienda "Las Lajas", de Izalco, y colindante con Coatepeque: "los jornaleros se escondían (...) Rogelio Hernández y Rogelio Escobar, eran agitadores de los sucesos de 1932" (Alvarenga 2006, p. 286).

La estancia o hacienda conocida como "Las Lajas”, de 34 caballerías y 142 cuadras (Montes, 1986, p.38) se conoce desde tiempos coloniales y abarca los municipios de Izalco, Armenia, hoy parte de El Congo, -antes Coatepeque-, departamento de Santa Ana y Sonsonate.

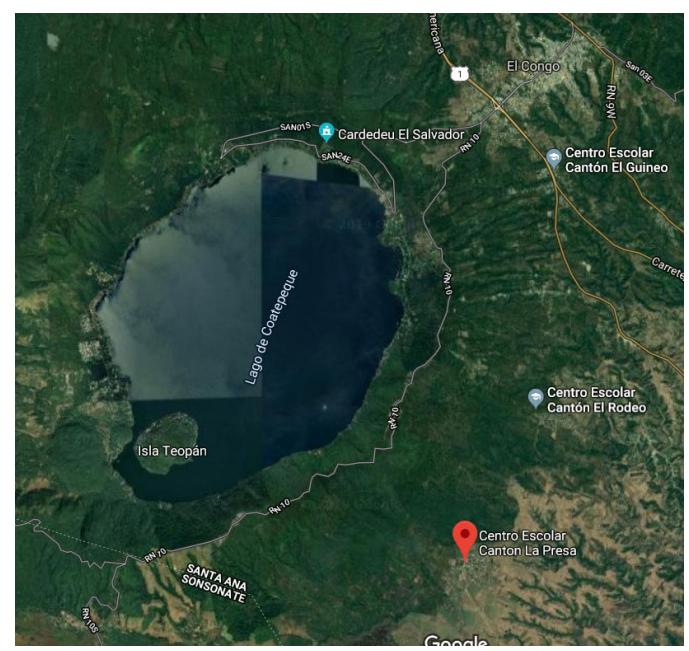

Imagen $n .^{\circ}$ 1. Haciendas Las Lajas Tomada de Google maps.

Por tener la amplitud y estar en los límites departamentales de Santa Ana y Sonsonate, probablemente se convirtió en una especie de refugio para diversos trabajadores y hasta, personas consideradas, por las autoridades, fuera de la ley. Es de recordar cómo Izalco,fue el foco de insurrección indígenacampesina de 1932. Así mismo, cuando se tomó el pueblo por los alzados, en enero de 1932, luego de que fueran enviadas tropas a repelerlos, "los indígenas huyeron a la zona norte del municipio, zona de cultivo de cafetales”(Leiva, 2011, p.24). Durante el siglo $\mathrm{XX}$, la hacienda Las Lajas "propiedad de las señoritas Barrientos”, “se repasta respetable cantidad de ganado y recoge abundante cosecha de maíz, frijol y cebolla (Barberena, 1998, p. 94). 
Pero será la hacienda "La Presa", vecina de "Las Lajas" la que se convertirá en un cantón del municipio de El Congo. Así, pasados los hechos del levantamiento indígena-campesino de 1932 se crea el municipio de El Congo, nombre que representa una irregularidad, en cuanto a toponimias locales salvadoreñas envueltas con nombre nabuat y una deidad católica. El 20 de marzo de 1933, escindiendo territorio de Coatepeque, se crea $\mathrm{El} \mathrm{Congo}{ }^{5}$.

Como se señaló anteriormente, la situación política y social era convulsa; entonces para propiciar el "orden” y el progreso por medio del cultivo del café, las autoridades, gobernadores, comandantes, comerciantes-civiles y el mismo general Martínez se ven inmiscuidos en la creación del municipio de El Congo.

\section{El control de la conflictividad}

Sin embargo, la interacción entre las poblaciones colindantes del -El Congo y Coatepeque-, no ha sido del todo armoniosa, hay y sigue existiendo disputas y rivalidades entre ambas poblaciones por procesos económicos, políticos y sociohistóricos, que seguramente tienen su base en elementos más profundos del pensamiento y las normas sociales, la cultura.

Como bien lo recoge la antropóloga Concepción de Guevara (1973), en El Congo se narran historias ridiculizando a la gente de Coatepeque $\mathrm{y}$ hacen notar que son conservadores y poco progresistas (p.75), ambas poblaciones no quieren saber la una de la otra.

5 Según, decreto n 31 D. O marzo de 1933
Esto permitió un clima de división y tensión y hasta el mismo cantón El Congo se dividió entre quienes querían que se elevara a municipio, y entre los que se oponían, o entre pequeños propietarios de parcelas y burgueses comerciantes especuladores. En esta lucha, los que estaban cerca del poder salieron con ventajas. Este punto es sensible de abordar pues, eran normas y valores institucionalizados por el "martinato". Habría que entender hasta dónde el General Hernández Martínez estaba comprometido con la "causa" de la creación de un nuevo municipio.

No obstante, el General Hernández Martínez tenía su rancho de descanso al borde del lago de Coatepeque (Gáleas, 2011, p. 11) y algunas crónicas periodísticas advierten de su interés por la creación del nuevo municipio de El Congo: "el señor presidente de la República, General Maximiliano Hernández Martínez, se interesó porque El Congo se convirtiera en pueblo" (Diario El Día, abril de 1933).

La conversión del cantón El Congo en municipio en 1933 se trató de un proceso económico asociado al cultivo de café; pero, también es un proceso político que buscaba legitimar la autoridad gubernamental -recién pasada la matanza de 1932-, controlar y pacificar la zona convulsa. No obstante, este proceso deja ver el sistema clientelar y la forma cómo procedía el "martinato".

Como bien lo demuestran los académicos (Ching, Tilley y López, 2007), el régimen martinista llevó a cabo lo que se denomina el sistema clientelar que establece relaciones jerárquicas basadas en el patrón-cliente. 
En el caso del presente trabajo, algunos documentos exponen cómo personajes ajenos al cantón El Congo pero ligados al poder salieron ganando con la creación del municipio, concretamente doctor Francisco Federico Reyes ${ }^{6}$ (1860-1951). Una nota periodística señala que “(para la creación del municipio) estuvieron también como arma activa el doctor Francisco Federico Reyes" (Diario El Día, abril de 1933) pero también según Castellanos (2002):

En el primer semestre de 1934 un grupo de profesionales terratenientes y empresarios, a cuya cabeza aparecieron los doctores Rodrigo Samayoa (excompañero de Martínez en la facultad de Derecho), Francisco A Reyes, y Atilio Pecorini aseguraron representar las "fuerzas vivas" del país, visitaron al Gral. Martínez, "maestro, estadista y militar", conocido "por su amor al país, su dedicación al trabajo y su honestidad" y le propusieron que "para que el trabajo realizado hasta la fecha continúe para el engrandecimiento de la nación, se postulara como candidato en los futuros comicios presidenciales. Al argumento de que esta postulación implicaría de hecho una reelección, contrapusieron el precedente de la candidatura y elección de Don Carlos Meléndez, en 1915 (p. 126).

Además, hay documentos de la historia del municipio de El Congo, que "agradecen" al doctor Francisco Antonio Reyes la donación de

6 Este abogado había sido funcionario de varios gobiernos; fue presidente y vicepresidente de la Asamblea legislativa varias veces en los años 30. Además, según documentos consultados en archivo nacional, algunas veces escribía su nombre como Francisco Antonio Reyes o Francisco A. Reyes; y en otras, como Francisco Federico Reyes o Franco. Fedo. Reyes, (estaba emparentado con el expresidente Tomas Regalado, 1899) e intentó correr a la presidencia. un predio que ahora ocupan la Alcaldía de El Congo, el mercado y la escuelita.

En relación con lo anterior, podemos establecer que había intereses en juego en la creación del municipio El Congo, mostrados a partir de acciones "favorables" a jefes y subordinados del martinato (Ching, 2007).

\section{Conclusiones}

Como se demuestra en el presente trabajo la frontera agrícola santaneca puso presión sobre los municipios de Coatepeque y, posteriormente parte de lo que sería el municipio de El Congo. Según Portillo (2006), se trató de pequeñas propiedades con algunos "beneficios de café" que, a su vez, se relacionaban con una burguesía agroexportadora santaneca. Este proceso no estuvo exento de atropellos, vejaciones y arbitrariedades.

Ciertamente para 1933, el contexto social era conflictivo: el General Hernández Martínez, tras un golpe de Estado en 1931, se hace del poder; también, en 1932 enfrenta un levamiento indígena-campesino con crudeza, que deja miles de muertos; antes y durante ese levantamiento hay huelgas, protestas, agitación política y persecución de los que el régimen considera sus enemigos.

A partir de estos hechos se crea una de las identidadesnacionalesbasada en el anticomunismo (Alvarenga, 2006), el Gobierno del "martinato" intervino con reformas económicas favorables a los grupos de poder, como la supresión de deudas y moratorias con los bancos particulares; tambiénimplementa una política clientelar que, entre otras cosas, permite la adhesión y fidelidad a su Gobierno y sus clientelas. 
En este sentido, es posible ver los intereses que se mezclan para conseguir la creación del municipio de El Congo (1933), aunado al hecho de que el mismo general Hernández Martínez tenía su rancho de descanso en el lago de Coatepeque -parte de lo que hoy es El Congo-; además, Hernández Martínez trastocó el poder municipal dando el visto bueno a los alcaldes y gobernadores. Por ejemplo, el primer alcalde de El Congo, con la venia del presidente, rigió los destinos del nuevo municipio por 13 años consecutivos.

Por otra parte, sectores ligados a las familias más influyentes política y económicamente estuvieron a la sombra del general Hernández Martínez, tal es el caso del abogado Francisco Antonio Reyes (emparentado con el general y expresidente Tomas Regalado) quien ejerció diferentes cargos estatales y se le consideraba uno de los principales caficultores en 1920 (Wilson 2004 p.61)

Como se constata en el trabajo fue el Dr. Francisco Antonio Reyes quien donó las tierras en el futuro pueblo de El Congo. Su figura representa al sector que tenía acceso al poder político, a los beneficios de la economía cafetalera y por tanto, dista mucho de las acciones de cualquier salvadoreño.

\section{Bibliografía}

Alvarenga, P. (2006). Cultura y ética de la violencia El Salvador 1880-1932. San Salvador: Dirección de Publicaciones e Impresos.

Anderson, T. (2006). El Salvador 1932 los sucesos politicos. San Salvador: Dirección de Publicaciones e Impresos.
Browning, D. (1998). El Salvador, la tierra y el hombre. San Salvador: Dirección de Publicaciones e Impresos.

Barberena, S. (1998). Monografías departamentales. San Salvador: Dirección de Publicaciones e Impresos.

Barón, R. (2002). La población de El Salvador $3^{a}$ edición. San Salvador: Dirección de Publicaciones e Impresos.

Castellanos, J. (2002). El Salvador 1930-1960 Antecedentes bistóricos de la guerra civil. San Salvador: Dirección de Publicaciones e Impresos.

Clará, C. (1973). Exploración etnográfica del departamento de Santa Ana. San Salvador: Ministerio de Educación.

Cañas, C. y Cortez, V. (2006). Historia del Órgano Legislativo de la República de El Salvador 19362006, tomo IV.San Salvador: Albacrome editores.

Ching, E.; Tilley, V.; López, C. (2007). Las masas, la matanza y el martinato en El Salvador. San Salvador: UCA Editores.

Escalante, P. (1992). Códice Sonsonate crónicas hispánicas. San Salvador: Dirección de Publicaciones e Impresos.

Gómez, I. (1992). Estadística general de la República de El Salvador (1858-1861). San Salvador: Concultura.

Gould, J. y Lauria, A. (2005, enero-diciembre). Nos llaman ladrones y se roban nuestro salario: hacia una interpretación de la movilización rural 
salvadoreña, 1929-1931 Revista Historia, San José, Costa Rica: n. ${ }^{\circ}$ 51-52, pp. 287-355.

Gómez, M. (2013, enero-marzo). Ser extranjero en Centroamérica. Génesis y evolución de las leyes de extranjería y migración en El Salvador: siglos XIX y XX. Revista Realidad, El Salvador, n. ${ }^{\circ} 135$, pp. 117- 145.

Gáleas, M. (2011). Sol y acero: la vida de don Guillermo Sol Bang. San Salvador: Editorial Cinco.

Lauria, A. (2003). Una república agraria los campesinos en la economía y la politica de El Salvador en el siglo XIX. San Salvador: Dirección de Publicaciones e Impresos.

Leiva, J. (2011). Los Izalcos: testimonio de un indigena. San Salvador: Imprenta Universitaria.
Montes, S. (1986). El agro salvadoreño. San Salvador: UCA Editores.

Portillo, G. (2006). La tenencia de la tierra en El Salvador La libertad 1897-1901, Santa Ana 1882-1884-1897-1898. San Salvador: Instituto de Estudios Históricos Antropológicos y Arqueológicos de la Universidad de El Salvador

Diario El Día. (5 de abril de 1993). Primeras noticias de nuestro corresponsal. Diario E1 Día.

Wilson, E. (2004). La crisis de integración nacional en El Salvador 1919-1935. San Salvador: Dirección de Publicaciones e Impresos. 